\title{
Foreword
}

\section{Preoperative Evaluation: Is It Time to View It as a Component of Perioperative Optimization?}

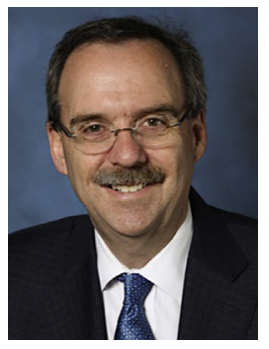

Lee A. Fleisher, MD Consulting Editor

The preoperative evaluation of the patient undergoing surgery has been the hallmark of the role of the anesthesiologists. Traditionally, the information was used to (1) discuss the risk of surgery as part of the decision to proceed and (2) provide a database upon which to make intraoperative decisions. With the development of the perioperative medicine movement, including Enhanced Recovery After Surgery (ERAS) and the Perioperative Surgical Home, there is an increasing number of strategies to "optimize" patients prior to surgery, including anemia clinics. While the evidence to support many of these strategies is limited, it makes physiologic sense. The issue includes articles outlining the development of a preoperative evaluation clinic and the implications of several comorbidities, such as cardiac disease, kidney disease, and diabetes. They have also included important new issues such as the assessment of frailty and cognitive function. In addition, the potential utility of genomics in the preoperative evaluation is discussed. It is, therefore, exciting that the two editors have created an issue that includes both the "traditional" approach and optimization strategies.

In identifying editors for this issue, I approached leaders of the Society for Perioperative Assessment and Quality Improvement (SPAQI). Zdravka Zafirova, MD is Associate Professor of Anesthesiology and Critical Care in the Department of Cardiovascular Surgery at Icahn School of Medicine and Mount Sinai Hospital system. She is the Director of Anesthesia Pre-operative Evaluation and Testing Center at Mount Sinai Hospital. She is a member of the Board of Directors of SPAQI and has expertise in the perioperative management of medically complicated patients. Richard D. Urman, MD, MBA, CPE, FASA is an anesthesiologist at the Brigham and Women's Hospital $(B W H)$ in Boston, Massachusetts and Associate Professor of Anesthesia at Harvard Medical School. He serves as Director of Anesthesia Services at the BWH Care Center 
at Chestnut Hill and Director (Anesthesia) of the interdepartmental Center for Perioperative Research at BWH and directs the newly established Perioperative Medicine Fellowship. Dr Urman is also treasurer of SPAQI. His research areas encompass patient outcomes, informatics, patient safety, novel anesthetic drugs, simulation, and operating room management. Together, they have created an important and informative issue.

Lee A. Fleisher, MD Perelman School of Medicine at University of Pennsylvania 3400 Spruce Street, Dulles 680 Philadelphia, PA 19104, USA

E-mail address: 